Arq. Bras. Med. Vet. Zootec., v.67, n.1, p.221-226, 2015

\title{
Atividade de nanoformulações de Melaleuca alternifolia e terpinen-4-ol em isolados de Rhodococcus equi
}

\author{
[Melaleuca alternifolia activity in nanoformulations and terpinen-4-ol \\ against Rhodococcus equi isolates] \\ L. Sagave, L.T. Gressler, F.C. Flores, C. B. Silva, A.P.C. Vargas, M. Lovato, \\ L.A. Sangioni, L. Pötter, S.A. Botton \\ Universidade Federal de Santa Maria - UFSM - Santa Maria, RS
}

\begin{abstract}
RESUMO
Rhodococcus equi é o agente etiológico da rodococose equina, importante doença respiratória de potros. Especialmente na última década, a emergência de cepas resistentes aos antimicrobianos empregados no tratamento da rodococose tem sido relatada. Nesse sentido, há a necessidade de estudos envolvendo terapias alternativas e novas tecnologias, incluindo o uso de plantas medicinais e nanotecnologia. Neste trabalho utilizou-se Melaleuca alternifolia nas seguintes formulações: óleo livre, nanocápsula, nanoemulsão e a combinação de óleo livre com nanocápsula e com nanoemulsão, além do seu composto majoritário, terpinen-4-ol, a fim de verificar a atividade antimicrobiana frente a isolados de $R$. equi de diferentes origens. Utilizou-se o método de microdiluição em caldo na determinação das concentrações inibitória mínima (CIM) e bactericida mínima (CBM) das diferentes formulações frente aos isolados $(\mathrm{n}=24)$. Verificou-se baixo potencial para atividade antibacteriana de $M$. alternifolia na formulação de óleo livre. Todavia, essa atividade foi potencializada quando se incorporou o óleo essencial às nanoformulações. $\mathrm{O}$ composto terpinen-4-ol demonstrou potencial atividade antibacteriana quando incorporado ao óleo essencial e quando utilizado isoladamente. Verificou-se que tanto M. alternifolia quanto terpinen-4-ol testados possuem atividade antimicrobiana contra isolados de $R$. equi, sugerindo seu emprego em estudos avaliando seu potencial para o tratamento da rodococose.
\end{abstract}

Palavras-chave: planta medicinal, nanotecnologia, rodococose, teste de susceptibilidade

\begin{abstract}
Rhodococcus equi causes rodococose in horses, characterized by bronchopneumonia in foals. Due to reports of antimicrobial resistance, it is important to develop studies involving alternative therapies and new technologies, including the use of medicinal plants and nanotechnology. In this work, the plant Melaleuca alternifolia in oil free formulations, nanocapsule, nanoemulsion and the combination of free and nanocapsule oil nanoemulsion, besides its major compound, terpinen-4-ol, were used in order to verify antimicrobial activity against isolates of $\mathrm{R}$. equi. The broth microdilution method was employed to determine the minimum inhibitory $(M I C)$ and minimum bactericidal $(M B C)$ concentrations of different formulations against 24 isolates. There was low antibacterial activity of M. alternifolia in oil free formulation; however, it was observed that the activity was enhanced when incorporated as essential oil the nanoformulations. The major compound, terpinen-4-ol, showed bactericidal and bacteriostatic activity when used alone. It is suggested that M. alternifolia, in association with nanocarriers systems, as well as terpinen-4-ol, presents potential for future studies concerning the equine rodococosis therapy.
\end{abstract}

Keywords: medicinal plant, nanotechnology, rodococosis, susceptibility test

Recebido em 8 de março de 2014

Aceito em 9 de setembro de 2014

E-mail: lauren.sagave@gmail.com 


\section{INTRODUÇÃO}

A rodococose equina é uma enfermidade infecciosa cosmopolita, e no Brasil é relatada como uma das mais importantes enfermidades respiratórias em potros (Ribeiro et al., 2005). O agente etiológico é a bactéria intracelular facultativa Rhodococcus equi, habitante do solo e saprófita do trato intestinal de herbívoros (Meijer e Prescott, 2004). A principal manifestação clínica da doença é a broncopneumonia piogranulomatosa, uma vez que a maioria dos animais contaminam-se pela inalação de partículas de poeira contendo $R$. equi (Muscatello et al., 2007).

O tratamento com antimicrobianos macrolídeos em associação com a rifampicina é a principal forma de controle da doença; no entanto, a existência de isolados de $R$. equi resistentes a esses compostos vem sendo relatada (Giguére et $a l .$, 2002). Devido à crescente preocupação com a resistência antimicrobiana observada, tanto na medicina humana quanto na veterinária, pesquisas envolvendo terapias alternativas, com plantas medicinais e seus compostos, vêm sendo realizadas, a fim de obter-se uma maior variedade de fármacos com ação antimicrobiana (Bertini et al., 2005). Devido aos inúmeros mecanismos de ação, em diferentes alvos da célula microbiana, manifestações de resistência ou adaptação microbiana aos vegetais medicinais e seus derivados raramente são relatadas (Bakkali et al., 2008). Apesar de todo esse potencial, no Brasil, somente cerca de $1 \%$ dos compostos fitoterápicos disponíveis comercialmente são desenvolvidos para uso em medicina veterinária (Ozaki e Duarte, 2006).

Melaleuca alternifolia, também conhecida como "árvore do chá", é uma planta nativa da Austrália, e o seu óleo essencial (extraído das folhas por hidrodestilação) possui comprovada ação antibacteriana, antifúngica e antiviral (Silva et al., 2002). Na composição do óleo de $M$. alternifolia estão presentes monoterpenos, sesquiterpenos e seus alcoóis correspondentes (Carson et al., 2006), sendo os seus principais constituintes: terpinen-4-ol (40\%), seguido do $\gamma$ terpineno $(23 \%)$ e $\alpha$-terpineno (10\%), destacando-se mais o efeito antimicrobiano do terpinen-4-ol (Oliva et al., 2003).
Estratégias visando aperfeiçoar as características físico-químicas de substâncias bioativas envolvem, também, inovações tecnológicas, como a nanotecnologia. Atualmente, sistemas coloidais incluindo as nanocápsulas, as nanoesferas, as nanoemulsões e outros sistemas nanoestruturados são alvos de pesquisas sobre novas formas de carrear fármacos para locais específicos e a liberação controlada dessas substâncias (Schaffazick et al., 2003). Nas áreas de produção e sanidade animal, relatos da utilização de nanoestruturas são escassos (Troncarelli et al., 2013). Como exemplo, um estudo experimental em microbiologia veterinária demonstrou a maior eficácia de estreptomicina e doxiciclina quando esses fármacos foram incorporados às nanoestruturas e testados frente a isolados de Brucella melitensis. Os autores concluíram que os fármacos livres foram menos eficazes na redução da carga bacteriana presente no fígado e no baço de $M u s$ musculus infectados do que na forma de nanocápsulas (Seleem et al., 2009). Mosqueira et al. (2010) patentearam nanocarreadores poliméricos do iodo para aplicação intramamária em ruminantes como uma alternativa para o tratamento de mastite, porém ainda se encontra em fase de estudos.

As nanoemulsões são dispersões de óleo em água, com as gotículas do óleo estabilizadas por tensoativos (Anton et al., 2008). As nanocápsulas possuem na sua composição a presença de um núcleo oleoso circundado por um fino invólucro polimérico, e o fármaco encontrase dissolvido no núcleo, adsorvido ou disperso na parede polimérica (Vauthier e Bouchemal, 2009). Esses sistemas submicrométricos são capazes de promover a diminuição de efeitos tóxicos e aumentar o índice terapêutico de fármacos (Schaffazick et al., 2003).

Devido aos crescentes relatos de resistência antimicrobiana de isolados de $R$. equi, e ao potencial antimicrobiano das plantas medicinais, especialmente em associação à nanotecnologia, este estudo teve como objetivo verificar a atividade antibacteriana de $M$. alternifolia, na forma de óleo livre e em sistemas nanoestruruados, bem como do seu composto majoritário, terpinen-4-ol, contra isolados de $R$. equi de diferentes origens. 


\section{MATERIAL E MÉTODOS}

O óleo essencial de $M$. alternifolia e o seu componente majoritário, terpinen-4-ol, foram obtidos comercialmente (Laszlo Aromaterapia, Belo Horizonte, Brasil, e Sigma Aldrich, São Paulo, Brasil, respectivamente). As nanoemulsões e nanocápsulas contendo $M$. alternifolia a $1 \%(10 \mathrm{mg} / \mathrm{mL})$ (Flores et al., 2011) foram preparadas pelos métodos de emulsificação espontânea (Bouchemal et al., 2004) e deposição interfacial do polímero préformado de poli ( $\varepsilon$-caprolactona) (Fessi et al., 1989), respectivamente, apresentando tamanho na faixa nanométrica (cerca de 200nm), potencial zeta negativo e índice de polidispersão abaixo de 0,25 .

Foram utilizados 24 isolados de $R$. equi, obtidos entre os anos de 1991 e 2012, provenientes de amostras de solo $(\mathrm{n}=8)$, fezes de animais hígidos $(n=8)$ e de animais com manifestações clínicas da rodococose $(n=8)$, coletados de 10 estabelecimentos de criação equina localizados na região sul do Brasil. Todos os isolados foram previamente identificados, fenotípica e genotipicamente, quanto ao gênero e espécie (Monego et al., 2009).

O perfil de susceptibilidade dos isolados de $R$. equi frente às diferentes formulações contendo óleo essencial de $M$. alternifolia foi avaliado pelo método de microdiluição em caldo MüellerHinton (MHC) (Himedia ${ }^{\circledR}$ Laboratories). Todos os isolados foram cultivados em Müeller-Hinton Ágar (MHA) (Himedia ${ }^{\circledR}$ Laboratories), em aerobiose a $37^{\circ} \mathrm{C}$, durante $24 \mathrm{~h}$. Para cada isolado, preparou-se um inóculo em solução salina $0,9 \%$, ajustado para a escala 0,5 de McFarland, posteriormente diluído em MHC e testado na concentração $1 \times 10^{5} \mathrm{UFC} / \mathrm{mL}$. As formulações testadas e suas combinações foram: 1. óleo essencial em dispersão grosseira ou óleo livre (OL), 2. Óleo essencial associado à nanocápsula (NC), 3. Óleo essencial associado à nanoemulsão (NE), 4. OL+NC (na combinação de $50 \%$ de cada um) e 5. OL+NE (na combinação de $50 \%$ de cada um). Adicionalmente, a atividade antimicrobiana do terpinen-4-ol foi avaliada frente aos isolados de $R$. equi. A concentração inibitória mínima (CIM) e concentração bactericida mínima (CBM) foram determinadas em conformidade com as diretrizes da Metodologia dos Testes de Sensibilidade a
Agentes Antimicrobianos por Diluição para Bactéria de Crescimento Aeróbico do Clinical Laboratory Standards Institute (Clinical and Laboratory Standards Institute, 2013). As concentrações das formulações de $M$. alternifolia testadas foram de $2.500 \mu \mathrm{g} / \mathrm{mL}$ a $1,2 \mu \mathrm{g} / \mathrm{mL}$, na razão 1:2. As concentrações do terpinen-4-ol variaram de $44.360 \mu \mathrm{g} / \mathrm{mL}$ a $21,660 \mu \mathrm{g} / \mathrm{mL}$. Todos os testes foram realizados em triplicata, incluindo os controles positivos e negativos.

Os valores de CIM e CBM para $R$. equi frente ao óleo essencial de $M$. alternifolia nas diferentes formulações foram avaliados com auxílio do teste de Kruskal-Wallis. Quando identificadas diferenças entre os tratamentos, aplicou-se o teste de Bonferroni para comparar as médias. Para avaliação dos resultados dos isolados frente ao terpinen-4-ol utilizou-se o teste do quiquadrado. As análises foram realizadas com auxílio do programa estatístico SAS, versão 9.2 (Statistical analysis system user's guide, 2001).

\section{RESULTADOS E DISCUSSÃO}

O óleo essencial de $M$. alternifolia na apresentação de OL não apresentou efeito antimicrobiano frente aos isolados clínicos e de solo de R. equi. Entretanto, 62,5\% (5/8) dos isolados de fezes foram inibidos nas concentrações de $2.500 \mu \mathrm{g} / \mathrm{mL}$ até $625 \mu \mathrm{g} / \mathrm{mL}$. Resultados de CIM de compostos de origem vegetal entre 1.500 e $600 \mu \mathrm{g} / \mathrm{ml}$ são considerados de efeito moderado; todavia, CIM acima de $1.600 \mu \mathrm{g} / \mathrm{mL}$ são considerados como fraca inibição (Aligianis et al., 2001). Dessa forma, pode-se inferir que o óleo essencial de $M$. alternifolia apresentou ação inibitória de fraca a moderada frente a isolados de fezes de $R$. equi. Estudos anteriores comprovaram a atividade bacteriostática e bactericida do óleo essencial de M. alternifolia sobre Porphyromonas gingivalis (Faria et al., 2012). Banes-Marshall et al. (2001) relataram que a maioria das bactérias são sensíveis ao óleo essencial de $M$. alternifolia na concentração de $10 \mathrm{mg} / \mathrm{mL}$; no entanto, Enterococcus faecalis e Pseudomonas aeruginosa necessitaram de concentrações maiores do óleo para serem inibidos. Neste estudo com $R$. equi, a maior concentração do óleo essencial na formulação de OL $(2.500 \mu \mathrm{g} / \mathrm{mL})$ não foi capaz de inibir a maioria (79,2\%) dos isolados de $R$. equi (19/24). 
As NE e NC contendo óleo essencial de $M$. alternifolia apresentaram efeito de inibição do crescimento de $100 \%$ (8/8) dos isolados de solo e isolados de fezes (8/8) de $R$. equi nas concentrações de $2.500 \mu \mathrm{g} / \mathrm{mL}$ até $312,5 \mu \mathrm{g} / \mathrm{mL}$, totalizando 16 isolados inibidos. Os isolados clínicos não foram inibidos nas concentrações de óleo essencial testadas. $\mathrm{O}$ uso das nanoestruturas contendo óleo essencial de $M$. alternifolia, em comparação ao OL, apresentou atividade inibitória 2,75 vezes maior $(\mathrm{P}<0.05)$. A associação de fármacos aos sistemas nanoestruturados, devido ao seu reduzido tamanho, propicia a melhora do índice terapêutico dos mesmos, como observado na atividade antimicrobiana da clorexidina associada à NC sobre Staphylococcus epidermidis, apresentando maior atividade em relação ao fármaco livre em um modelo experimental ex vivo com pele de orelha de suíno (Lboutounne et al., 2002). Paulo et al. (2010) avaliaram a atividade antifúngica de nanopartículas contendo anfotericina $\mathrm{B}$, obtendo resultados satisfatórios em comparação ao fármaco livre. Melhora da atividade inibitória do óleo essencial de $M$. alternifolia, quando associado a nanocarreadores, também foi demonstrada por Flores et al. (2013) frente ao fungo dermatófito, em modelo experimental de infecção ungueal, quando comparado à atividade do OL. No presente experimento, o OL e as nanoestruturas contendo óleo essencial não apresentaram efeito inibitório do crescimento de $R$. equi após o seu plaqueamento em MHA.

Diante da possibilidade de ocorrer um efeito complementar entre o OL de $M$. alternifolia e as nanoestruturas contendo óleo essencial, avaliouse a atividade antimicrobiana das combinações de $\mathrm{OL}+\mathrm{NE}$ e $\mathrm{OL}+\mathrm{NC}$. Com isso, observou-se atividade antimicrobiana em $100 \%$ (24/24) dos isolados, sem haver diferença estatística entre as associações. Acredita-se que esse resultado esteja relacionado à dose inicial de ataque, oferecida pelo OL, e a manutenção desta pelo controle da liberação do óleo proporcionado pelas nanoestruturas (Flores et al., 2011). O efeito antimicrobiano do óleo ocorreu imediatamente após a sua aplicação, possivelmente devido ao contato direto com a membrana do micro-organismo, provocando alterações na permeabilidade da membrana e inibição da respiração celular, conforme sugerido por Carson et al. (2006). Além disso, os sistemas nanoestruturados promovem a liberação gradual dos compostos, aumentando o tempo de contato e, por apresentarem reduzido tamanho, proporcionam a deposição de um maior número de partículas na superfície da célula microbiana, expondo maior quantidade da substância ativa (Lboutounne et al., 2002). Dessa forma, concluise que tanto a formulação de $\mathrm{NC}$ como de $\mathrm{NE}$, contendo o óleo essencial de $M$. alternifolia em associação ao OL, possuem atividade antibacteriana contra isolados de $R$. equi de diferentes origens.

O terpinen-4-ol é um dos principais responsáveis pela atividade antimicrobiana do óleo essencial de $M$. alternifolia, representando aproximadamente $40 \%$ do óleo essencial (Carson et al., 2006). Com o terpinen-4-ol utilizado isoladamente frente aos isolados de $R$. equi, obteve-se CIM nas concentrações entre $11.090 \mu \mathrm{g} / \mathrm{mL}$ e $5.545 \mu \mathrm{g} / \mathrm{mL}$ e CBM nas concentrações de $44.360 \mu \mathrm{g} / \mathrm{mL}$ a $11.090 \mu \mathrm{g} / \mathrm{mL}$, demonstrando que a atividade antibacteriana do terpinen-4-ol isolado foi 18,02 vezes menos efetiva que na composição do óleo essencial de M. alternifolia. Em contrapartida, o terpinen-4-ol demonstrou superior capacidade antibacteriana quando utilizado isoladamente do que quando incorporado ao óleo essencial de M. alternifolia frente a isolados de Escherichia coli e Staphylococcus aureus (Cox et al., 2001) Da mesma forma, HAMMER et al. (2003) constataram que o terpinen-4-ol isolado foi mais eficaz que o óleo essencial da planta frente à levedura Candida albicans. A estrutura celular e metabolismo específico do $R$. equi podem ter determinado a menor ação do terpinen-4-ol sobre esse agente, quando comparado com os microorganismos anteriormente descritos.

Considerando a problemática no tratamento da rodococose equina, a resistência antimicrobiana ao tratamento convencional e de posse dos resultados obtidos, o óleo essencial da planta $M$. alternifolia, especialmente associado a sistemas nanoestruturados, possui potencial uso como agente inibitório frente a isolados de $R$. equi. 


\section{CONCLUSÃO}

O óleo essencial da planta $M$. alternifolia apresenta atividade antibacteriana frente a isolados de $R$. equi, e essa atividade é fortemente potencializada diante da associação com a NC e NE. Terpinen-4-ol apresenta atividade antibacteriana frente a isolados de $R$. equi, porém sua utilização de forma isolada não foi superior ao uso do óleo essencial da planta. Devido aos relatos de resistência antimicrobiana de isolados de $R$. equi, estudos farmacológicos devem ser conduzidos a fim de avaliar a ação terapêutica de compostos contendo óleo essencial de $M$. alternifolia como uma alternativa ao tratamento convencional da rodococose equina.

\section{REFERÊNCIAS}

ALIGIANIS, N.; KALPOUTZAKIS, E.; MITAKU, S. et al. Composition and antimicrobial activity of the essential oil of two Origanum species. J. Agric. Food Chem., v.49, p.4168-4170, 2001.

ANTON, N.; BENOIT, J.; SAULNIER, P. et al. Design and production of nanoparticles formulated from nano-emulsion templates - A review. J. Control. Releas., v.128, p.185-199, 2008.

BAKKALI, F.; AVERBECK, S.; AVERBECK, D. et al. Biological effects of essential oils - A review. Food. Chem. Toxicol., v.46, p.446-475, 2008 .

BANES-MARSHALL， L.P.; CAWLEY， P.; PHILLIPS, C.A. In vitro activity of Melaleuca alternifolia (tea tree) oil against bacterial and Candida spp. Isolates from clinical specimens. Br. J. Biomed. Sci., v.58, p.139-145, 2001.

BERTINI, L.M.; PEREIRA, A.F.; OLIVEIRA, C.L.L. et al. Perfil de sensibilidade de bactérias frente a óleos essenciais de algumas plantas do nordeste do Brasil. Infarm., v.17, p.80-83, 2005.

BOUCHEMAL, K.; BRIANCON, S.; PERRIER, E. et al. Nano-emulsion formulation using spontaneous emulsification: solvent, oil and surfactant optimization. Int. J. Pharm., v.51, p.280-241, 2004.

CARSON, C.F.; HAMMER, K.A.; RILEY, T.V. Melaleuca alternifolia (Tea Tree) Oil: a review of antimicrobial and other medicinal properties. Clin. Microbiol. Rev., v.19, p.50-62, 2006.
CLINICAL And Laboratory Standards Institute (CLSI). Metodologia dos Testes de Sensibilidade a Agentes Antimicrobianos por Diluição para Bactéria de Crescimento Aeróbico. Pensilvânia:Wayne, 2013. 53p.

COX, S.D.; MAN, C.M.; MARKHAM, J.L. et al. The mode of antimicrobial action of the essential oil of Melaleuca alternifolia (tea tree oil). J. Appl. Microbiol., v.88, p.170-175, 2000

FARIA, T.P.M.; GRAZIANO, T.S.; CALIL, C.M. et al. Atividade do óleo de Melaleuca alternifolia sobre o crescimento e expressão gênica de Porphyromonas gingivalis. J. Clin. Periodonto., v.22, p.80-86, 2012.

FESSI, H.; PUISIEUX, F.; DEVISSAGUET, J.P. et al. Nanocapsule formation by interfacial polymer deposition following solvent displacement. Int. J. Pharm., v.55, p.1-4, 1989.

FLORES F.C.; LIMA, J.A.; RIBEIRO, R.F. et al. Antifungal activity of nanocapsule suspensions containing Tea Tree oil on the growth of Trichophyton rubrum. Mycopathol., v.175, p.1-2, 2013.

FLORES F.C.; RIBEIRO, R.F.; OURIQUE, A.F. et al. Nanostructured systems containing an essential oil: protection against volatilization. Quím. Nov., v.34, p.968-972, 2011.

GIGUÉRE, S.; GASKIN, J.M.; MILLER, C. et al. Evaluation of a commercially available hyperimmune plasma product for prevention of naturally acquired pneumonia caused by Rhodococcus equi in foals. J. Am. Vet. Med. Assoc., v.220, p.59-63, 2002.

HAMMER, K.A.; CARSON, C.F.; RILEY, T.V. Antifungal activity of the components of Melaleuca alternifolia (tea tree) oil. J. Appl. Microbiol., v.95, p.853-860, 2003.

LBOUTOUNNE, H.; CHAULET, J.; PLOTON, C. et al. Sustained ex vivo skin antiseptic activity of chlorhexidine in poly (e-caprolactone) nanocapsule encapsulated form and as a digluconate. J. Control. Releas., v.82, p.319-334, 2002.

MEIJER, W.G.; PRESCOTT, J.F. Rhodococcus equi. Vet. Res., v.35, p.383-396, 2004. 
MONEGO, F.; MABONI, F.; KREWER, C. et al. Molecular characterization of Rhodococcus equi from horse breeding farms by means of multiplex PCR for the vap gene family. Curren. Microb., v.58, p.399-403, 2009.

MOSQUEIRA, V.C.F.; ARAÚJO, R.S.; BRANDÃO, H.M. et al. Composição nanoparticulada contendo antibióticos para administração intramamária de uso animal. Patente no: WO 2011150481 A1, 2010.

MUSCATELLO, G.; LEADON, D.P.; KLAY, M. et al. Rhodococcus equi infection in foals: the science of 'rattles'. Equine. Vet. J., v.39, p.470478, 2007.

OLIVA, B.; PICCIRILLI, E.; CEDDIA, T. et al. Antimycotic activity of Melaleuca alternifolia essential oil and its major components. Lett. Appl. Microbiol., v.37, p.185-187, 2003.

OZAKI, A.T.; DUARTE, P.C. Fitoterápicos utilizados na medicina veterinária, em cães e gatos. Infarm., v.18, p.17-25, 2006.

PAULO, C.S.O.; VIDAL, M.; FERREIRA, L.S. Antifungal nanoparticles and surfaces. Biomacromol., v.11, p.2810-2817, 2010.

RIBEIRO, M.G.; SEKI, I.; YASUOKA, K. et al. Molecular epidemiology of virulent Rhodococcus equi from foals in Brazil: virulence plasmids of 85-kb type I, 87-kb type I, and a new variant, 87-kb type III. Comp. Immunol. Microb., v.28, p.53-61, 2005.
STATISTICAL analysis system user's guide SAS: statistics. Version 9.2, Cary: Statistical Analysis System Institute, 2001. 1686p.

SCHAFFAZICK, R.S.; GUTERRES, S.S.; FREITAS, L.L. et al. Caracterização e estabilidade físico-química de sistemas poliméricos nanoparticulados para administração de fármacos. Quím. Nov., v.26, p.726-737, 2003.

SELEEM, M.N.; JAIN, N.; POTHAYEE, N. et al. Targeting Brucella melitensis with polymeric nanoparticles containing streptomycinand doxycycline. FEMS Microbiol., v.294, p.24-31, 2009.

SILVA, R.S.S.; DEMUNER, A.J.; BARBOSA, L.C.A. et al. Efeito do estresse hídrico sobre características de crescimento e a produção de óleo essencial de Melaleuca alternifolia Cheel. Acta. Sci. Agron., v.24, p.363-368, 2002.

TRONCARELLI, M.Z.; BRANDÃO, H.M.; GERN, J.C. et al. Mastite bovina sob nanocontrole: A própolis nanoestruturada como nova perspectiva de tratamento para rebanhos leiteiros orgânicos. Vet. Zootec., v.20, p.124-136, 2013.

VAUTHIER, C.; BOUCHEMAL, K. Methods for the Preparation and Manufacture of Polymeric Nanoparticles. Pharmac. Res., v.26, p.726-737, 2009. 